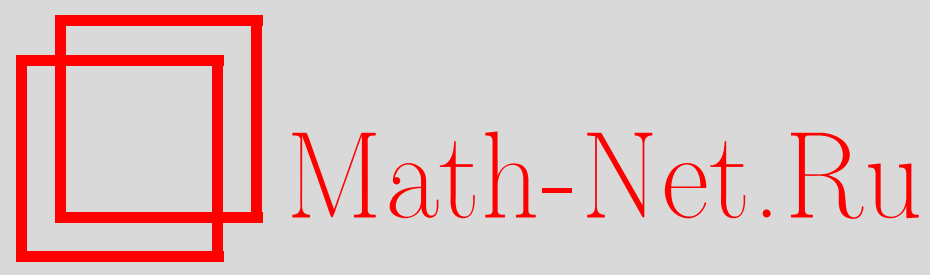

Ю. Н. Бибиков, Сохранение и бифуркация инвариантного тора векторного поля, Матем. заметки, 1997, том 61, выпуск $1,34-44$

DOI: https://doi.org/10.4213/mzm1480

Использование Общероссийского математического портала Math-Net.Ru подразумевает, что вы прочитали и согласны с пользовательским соглашением http://www . mathnet.ru/rus/agreement

Параметры загрузки:

IP : 54.162 .127 .20

26 апреля 2023 г., 13:52:02

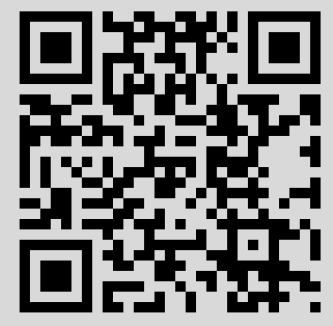




\section{СОХРАНЕНИЕ И БИФУРКАЦИЯ ИНВАРИАНТНОГО ТОРА ВЕКТОРНОГО ПОЛЯ}

\section{Ю. Н. Бибиков}

Рассматриваются характеризуемые малым параметром $\varepsilon \geqslant 0$ малые возмущения гладкого векторного поля в $\mathbb{R}^{n+m}$, имеющего инвариантный тор $T^{m}$. Предполагается, что поток на торе $T^{m}$ квазипериодический с $m$ базисными частотами, удовлетворяюшими определенным условиям диофантового типа, а матрица $\Omega$ коэффициентов системы уравнений в вариациях относительно инвариантного тора постоянна.

Исследуется вопрос о существовании при $\varepsilon>0$ инвариантных торов различных размерностей в случае, когда $\Omega$ - неособая матрица, допускающая чисто мнимые собственные числа.

Библиограффия: 8 названий.

1. Постановка задачи. Пусть $L$-гладкое векторное поле на $\mathbb{R}^{n+m}, i$ - вложение в $\mathbb{R}^{n+m}$ тора $T^{m}=\left\{\left(\theta_{1}, \ldots, \theta_{m}\right) \operatorname{modd} 2 \pi\right\}$. Если подмногообразие $i T^{m}$ инвариантно относительно поля $L$, мы будем назьвать его $m$-мерным инвариантным тором и обозначать по-прежнему $T^{m}$.

Предположим, что в окрестности тора $T^{m}$ в $\mathbb{R}^{n+m}$ можно ввести координаты $\theta=$ $\left(\theta_{1}, \ldots, \theta_{n}\right), x=\left(x_{1}, \ldots, x_{n}\right)$ так, чтобы уравнение $T^{m}$ имело вид $x=0$ и чтобы при этом выполнялось условие: если в координатах $\theta, x$ поле $L$ задается с помощью формулы

$$
L=\sum_{i=1}^{m} F_{i}(\theta, x) \frac{\partial}{\partial \theta_{i}}+\sum_{j=1}^{n} G_{j}(\theta, x) \frac{\partial}{\partial x_{j}}
$$

то в системе уравнений относительно тора $T^{m}$

$$
\dot{\theta}=F(\theta, 0), \quad \dot{x}=\frac{\partial G}{\partial x}(\theta, 0) x
$$

функции $F(\theta, 0),(\partial G / \partial x)(\theta, 0)$ не зависят от $\theta\left(F_{i}, G_{j}\right.$ - координатные функции векторов $F, G)$. Положим $F(\theta, 0)=\omega,(\partial G / \partial x)(\theta, 0)=\Omega$.

Рассмотрим возмущение векторного поля $L$, характеризуемое малым параметром $\varepsilon \geqslant 0$. Пусть возмущенная система дифференциальных уравнений имеет вид

$$
\dot{\theta}=\omega+\Theta(\theta, x, \varepsilon), \quad \dot{x}=\Omega x+X(\theta, x, \varepsilon),
$$

Работа выполнена при частичной поддержке Российского фонда фундаментальных исследований, грант № 94-01-1717, и Международного научного фонда, грант NUX000. 
где $\Theta(\theta, 0,0)=0, X(\theta, 0,0)=0,(\partial X / \partial x)(\theta, 0,0)=0$, а функции $\Theta, X$ - достаточно гладкие при $\|x\|<x^{*}, 0 \leqslant \varepsilon<\varepsilon^{*}, \theta \in T^{m}$.

Мы рассматриваем вопрос о сохранении и бифуркации инвариантного тора $T^{m}$ при $\varepsilon>0$ в случае, когда матрица $\Omega$ в системе (1) является неособой, но допускает чисто мнимые собственные числа.

Как мы увидим в дальнейшем, при определенных условиях ответы на поставленные вопросы не зависят от членов разложения функций $\Theta, X$ по степеням $\varepsilon, x$ вьше определенного порядка. Такие случаи, следуя А. М. Ляпунову, будем назьвать алгебраическими. Случаи, когда необходимо принять во внимание все члены указанных разложений, назьваем трансцендентными.

2. Сохранение инвариантного тора в алгебраических случаях. Как показал Н. Н. Боголюбов (см [1]), если $\Omega$ - некритическая матрица, т.е. если $\Omega$ не имеет чисто мнимых собственных чисел, то система (1) имеет при достаточно малых $\varepsilon>0$ инвариантный $m$-мерньй тор $T_{\varepsilon}^{m} \rightarrow T^{m}$ при $\varepsilon \rightarrow 0$. Предположим, что матрица $\Omega$ имеет чисто мнимые собственные числа $\lambda_{1}, \ldots, \lambda_{2 l}$, не равные нулю и друг другу, причем остальные собственные числа $\lambda_{2 l+1}, \ldots, \lambda_{n}$ не лежат на мнимой оси.

Если положить $P=\operatorname{diag}\left(\lambda_{1}, \ldots, \lambda_{2 l}\right)$, то в подходящих координатах систему (1) можно представить в виде

$$
\begin{aligned}
& \dot{\theta}=\omega+\Theta(\theta, \xi, \eta, \varepsilon), \\
& \dot{\xi}=P \xi+\Xi(\theta, \xi, \eta, \varepsilon), \\
& \dot{\eta}=Q \eta+Y(\theta, \xi, \eta, \varepsilon),
\end{aligned}
$$

где при $\varepsilon=\xi=\eta=0 \Xi=Y=\Theta=0, \partial \Xi / \partial(\xi, \eta)=\partial Y / \partial(\xi, \eta)=0, Q$ - некритическая матрица, уравнения, соответствуюшие комплексно сопряженным $\lambda_{j}, j=1, \ldots, 2 l$, комплексно сопряжены.

Предположим, что частоты $\omega_{i}, i=1, \ldots, m$, образующие вектор $\omega$, и числа $\lambda_{j}=i \alpha_{j}$, $j=1, \ldots, 2 l$, удовлетворяют диофантовому условию

$$
\left|\sum_{i=1}^{m} q_{i} \omega_{i}+\sum_{j=1}^{2 l} p_{j} \alpha_{j}\right|>\gamma|q|^{-\tau}, \quad \gamma>0, \quad \tau>0
$$

где $q_{i}, p_{j}$ - целые числа, такие, что $|q|=\left|q_{1}\right|+\cdots+\left|q_{m}\right|>0,|p|=\left|p_{1}\right|+\cdots+\left|p_{2 l}\right| \leqslant 2$, $\left|p_{1}+\cdots+p_{2 l}\right| \leqslant 1$.

ЛЕмма 1. При выполнении условия (3) для любого натурального р существует замена

$$
\begin{aligned}
& \theta=\varphi+\varepsilon f(\varphi, \varepsilon), \\
& \xi=u+\varepsilon g(\varphi, u, \varepsilon), \\
& \eta=v+\varepsilon h(\varphi, u, \varepsilon),
\end{aligned}
$$

әде $\varphi \in T^{m}, g, h$ - линейные функиии переменной $u$ (координаты вектора $и$, соответствуюшие комплексно сопряхенным координатам вектора $\xi$, тохе комп- 
лексно сопряжень), которая приводит систему (2) к виду

$$
\begin{aligned}
& \dot{\varphi}=\omega+\sum_{i=1}^{\nu} \varepsilon^{i} a_{i}+\Phi(\varphi, u, v, \varepsilon), \\
& \dot{u}=\left(P+\sum_{i=1}^{\nu} \varepsilon^{i} B_{i}\right) u+U(\varphi, u, v, \varepsilon), \\
& \dot{v}=Q v+V(\varphi, u, v, \varepsilon),
\end{aligned}
$$

әде $a_{i}$ - постоянные векторы, $B_{i}$ - постоянные диагональные матрицы, при $u=v=0$

$$
\begin{gathered}
\Phi=O\left(\varepsilon^{\nu+1}\right), \quad U=O\left(\varepsilon^{2 \nu+1}\right), \quad V=O\left(\varepsilon^{2 \nu+1}\right), \\
\frac{\partial U}{\partial u}=O\left(\varepsilon^{\nu+1}\right), \quad \frac{\partial V}{\partial u}=O\left(\varepsilon^{\nu}\right) .
\end{gathered}
$$

ДокАЗАТЕЛЬСтво. Замену (4) ищем в виде

$$
\begin{aligned}
& \theta=\varphi+\sum_{i=1}^{\nu} \varepsilon^{i} f_{i}(\varphi) \\
& \xi=u+\sum_{i=1}^{2 \nu} \varepsilon^{i} g_{i}(\varphi)+\left(\sum_{i=1}^{\nu} \varepsilon^{i} G_{i}(\varphi)\right) u \\
& \eta=v+\sum_{i=1}^{2 \nu} \varepsilon^{i} h_{i}(\varphi)+\left(\sum_{i=1}^{\nu-1} \varepsilon^{i} H_{i}(\varphi)\right) u
\end{aligned}
$$

где $G_{i}, H_{i}$ - матричные функции. Дифференцируя эти равенства по $t$ и учитьвая $(2)$ и (5), получаем равенства

$$
\begin{aligned}
\omega+\Theta(\varphi+\varepsilon f, u+\varepsilon g, v+\varepsilon h, \varepsilon)=\omega+\sum_{i=1}^{\nu} \varepsilon^{i} a_{i}+\Phi+\sum_{i=1}^{\nu} \varepsilon^{i} \frac{\partial f_{i}}{\partial \varphi}\left(\omega+\sum_{i=1}^{\nu} \varepsilon^{i} a_{i}+\Phi\right) \\
P\left(u+\sum_{i=1}^{2 \nu} \varepsilon^{i} g_{i}+\left(\sum_{i=1}^{\nu} \varepsilon^{i} G_{i}\right) u\right)+\Xi(\varphi+\varepsilon f, u+\varepsilon g, v+\varepsilon h, \varepsilon) \\
=\left(P+\sum_{i=1}^{\nu} \varepsilon^{i} B_{i}\right) u+U+\sum_{i=1}^{2 \nu} \varepsilon^{i} \frac{\partial g_{i}}{\partial \varphi}\left(\omega+\sum_{i=1}^{\nu} \varepsilon^{i} a_{i}+\Phi\right) \\
+\sum_{i=1}^{\nu} \varepsilon^{i}\left[\frac{\partial G_{i}}{\partial \varphi}\left(\omega+\sum_{i=1}^{\nu} \varepsilon^{i} a_{i}+\Phi\right)\right] u+\sum_{i=1}^{\nu} \varepsilon^{i} G_{i}\left(P u+\sum_{i=1}^{\nu} \varepsilon^{i} B_{i} u+U\right) \\
Q\left(v+\sum_{i=1}^{2 \nu} \varepsilon^{i} h_{i}+\left(\sum_{i=1}^{\nu-1} \varepsilon^{i} H_{i}\right) u\right)+Y(\varphi+\varepsilon f, u+\varepsilon g, v+\varepsilon h, \varepsilon) \\
=Q v+V+\sum_{i=1}^{2 \nu} \varepsilon^{i} \frac{\partial h_{i}}{\partial \varphi}\left(\omega+\sum_{i=1}^{\nu} \varepsilon^{i} a_{i}+\Phi\right) \\
+\sum_{i=1}^{\nu-1} \varepsilon^{i}\left[\frac{\partial H_{i}}{\partial \varphi}\left(\omega+\sum_{i=1}^{\nu} \varepsilon^{i} a_{i}+\Phi\right)\right] u+\sum_{i=1}^{\nu} \varepsilon^{i} H_{i}\left(P u+\sum_{i=1}^{\nu} \varepsilon^{i} B_{i} u+U\right) .
\end{aligned}
$$


В этих равенствах используется следующее обозначение: если $A$ - матричная функция переменной $\varphi, b$ - вектор-столбец, то

$$
\frac{\partial A}{\partial \varphi} b=\sum_{k=1}^{m} \frac{\partial A}{\partial \varphi_{k}} b_{k}
$$

где $\varphi_{k}, b_{k}-$ координаты векторов $\varphi, b$ соответственно.

Полагаем во всех трех равенствах (7) $u=v=0$ и приравниваем коэффициенты при степенях $\varepsilon$ от 1 до $\nu$ в первом равенстве и от 1 до $2 \nu$ во втором и третьем равенствах. Далее полагаем во втором и третьем равенствах $v=0$ и, рассматривая только линейную по $u$ часть, приравниваем коэффищиенты при степенях $\varepsilon$ от 1 до $\nu$ во втором равенстве и от 1 до $\nu-1$ в третьем. В результате для определения функций $f_{i}, g_{i}, h_{i}, G_{i}, H_{i}$ получим уравнения

$$
\begin{aligned}
& \frac{\partial f_{i}}{\partial \varphi} \omega=r_{i}(\varphi)-a_{i}, \quad \frac{\partial g_{i}}{\partial \varphi} \omega-P g_{i}=s_{i}(\varphi), \quad \frac{\partial h_{i}}{\partial \varphi} \omega-Q h_{i}=\sigma_{i}(\varphi), \\
& \frac{\partial G_{i}}{\partial \varphi} \omega+G_{i} P-P G_{i}=S_{i}(\varphi)-B_{i}, \quad \frac{\partial H_{i}}{\partial \varphi} \omega+H_{i} P-Q H_{i}=T_{i}(\varphi),
\end{aligned}
$$

где $r_{i}, s_{i}, \sigma_{i}, S_{i}, T_{i}$ - известные функции $\varphi$, если $f_{k}, g_{k}, h_{k}, G_{k}, H_{k}$, у которых $k<i$, уже определены. В качестве $a_{i}$ выбираем средние значения функций $r_{i}(\varphi)$, а в качестве $B_{i}-$ диагональные матрицы со средними значениями матрищ $S_{i}(\varphi)$ на диагоналях. Тогда при условиях (3) эти уравнения разрешимы в классепериодических по координатам вектора $\varphi$ функций (см. [1], [2]). Лемма доказана.

Изменим масштаб измерения переменных $u, v$ :

$$
u=\varepsilon^{\nu+1 / 2} z, \quad v=\varepsilon^{2 \nu} w .
$$

В силу (6) система (5) примет вид

$$
\begin{aligned}
& \dot{\varphi}=\omega+\sum_{i=1}^{\nu} \varepsilon^{i} a_{i}+\varepsilon^{\nu+1 / 2} \Phi^{*}(\varphi, z, w, \sqrt{\varepsilon}), \\
& \dot{z}=\left(P+\sum_{i=1}^{\nu} \varepsilon^{i} B_{i}\right) z+\varepsilon^{\nu+1 / 2} Z(\varphi, z, w, \sqrt{\varepsilon}), \\
& \dot{w}=Q w+\sqrt{\varepsilon} W(\varphi, z, w, \sqrt{\varepsilon}),
\end{aligned}
$$

где $\Phi^{*}, Z, W$ - гладкие функции при малых $\|z\|,\|w\|, \sqrt{\varepsilon}$ и $\varphi \in T^{m}$.

Положим $B_{i}=\operatorname{diag}\left(B_{1}^{(i)}, \ldots, B_{2 l}^{(i)}\right)$. Справедлива

ТЕОрема 1. Если выполнено условие (3) и существует натуральное $\nu$, такое, чmo

$$
\left|\operatorname{Re} B_{k}^{(1)}\right|+\cdots+\left|\operatorname{Re} B_{k}^{(\nu)}\right|>0, \quad k=1, \ldots, 2 l,
$$

то система (1) имеет при достаточно малых $\varepsilon>0$ инвариантный тор $T_{\varepsilon}^{m} \rightarrow T^{m}$ $n p u \varepsilon \rightarrow 0$. 
ДокАЗАтЕльСтво. В силу условия (10) матрица $P+\sum_{i=1}^{\nu} \varepsilon^{i} B_{i}$ является диагональной с блоками вида $i C(\varepsilon)+\varepsilon^{r} R(\varepsilon)$, где $0<r \leqslant \nu, C(\varepsilon), R(\varepsilon)$ - вещественные диагональные матрицы, причем $R(0)$ - матрища с ненулевыми диагональными элементами. Пусть число таких блоков равно $\sigma$. Систему (9) можно записать в виде системы

$$
\begin{aligned}
\dot{\varphi} & =\omega+\sum_{i=1}^{\nu} \varepsilon^{i} a_{i}+\varepsilon^{\nu+1 / 2} \Phi^{*}, \\
\dot{z}_{k} & =\varepsilon^{r_{k}}\left(i C_{k}(\varepsilon) \varepsilon^{-r_{k}}+R_{k}(\varepsilon)\right) z_{k}+\varepsilon^{\nu+1 / 2} Z_{k}, \\
\dot{w} & =Q w+\sqrt{\varepsilon} W,
\end{aligned}
$$

где $0<r_{k} \leqslant \nu$. Не нарушая общности, считаем, что $R_{k}=\operatorname{diag}\left(R_{k}^{+}, R_{k}^{-}\right)$, где диагональные элементы $R_{k}^{+}$положительны, а диагональные элементы $R_{k}^{-}$отрицательны. Пусть соответственно $\varepsilon^{-r_{k}} C_{k}=\operatorname{diag}\left(C_{k}^{(1)}, C_{k}^{(2)}\right)$. Образуем матричные функции

$$
J_{k}(t)=\left\{\begin{array}{rc}
-\operatorname{diag}\left(e^{-\Lambda_{k}^{+} t}, 0\right) & \text { при } t>0, \\
\operatorname{diag}\left(0, e^{-\Lambda_{k}^{-}}\right) & \text {при } t<0,
\end{array}\right.
$$

где $\Lambda_{k}^{+}=\left(i C_{k}^{(1)}+R_{k}^{+}\right), \Lambda_{k}^{-}=\left(i C_{k}^{(2)}+R_{k}^{-}\right), k=1, \ldots, \sigma$. При всех $t \in \mathbb{R}$ справедлива оценка

$$
\left\|J_{k}(t)\right\| \leqslant \beta e^{-\alpha t}, \quad \alpha>0, \quad \beta>0 .
$$

Используя эту оценку, точно так же, как при доказательстве леммы 2.1 работы [4] (см. также [2], [3]), докажем, что система (9) имеет интегральное многообразие $z=z(\varphi, \sqrt{\varepsilon}), w=w(\varphi, \sqrt{\varepsilon})$. учитьвая произведенное изменение масштаба переменных и замену (4), получаем утверждение теоремы.

Поскольку матрица $\Omega$ допускает чисто мнимые собственные числа, естественно ожидать, что наряду с построенным $m$-мерным инвариантным тором $T_{\varepsilon}^{m}$ существует и тор большей размерности. Этот вопрос мы рассматриваем в следующем пункте.

3. Бифуркация инвариантного тора в алгебраических случаях. Рассмотрим систему (5), правые части которой удовлетворяют условиям (6). Вьполним еще одну замену переменных

$$
\varphi=\psi+F(\psi, z, \varepsilon), \quad u=z+G(\psi, z, \varepsilon), \quad v=x+H(\psi, z, \varepsilon),
$$

где $\psi \in T^{m}, F, G, H$ - полиномы по координатам $u, F=O(|u|), G=O\left(|u|^{2}\right), H=O\left(|u|^{2}\right)$. В результате получим систему того же вида, что и (5), с заменой переменных $\varphi, u, v$ на $\psi, z, x$ соответственно. При этом условие (6) сохраняет силу. Однако, используя нормальную форму системы, подбором функций $F, G, H$ можно произвести дальнейшее упрощение системы.

Положим в системе $(5) u=(y, \bar{y})$, где $y-l$-вектор, черта обозначает комплексную сопряженность. Используя обозначение $y \cdot \bar{y}=\left(y_{1} \bar{y}_{1}, \ldots, y_{l} \bar{y}_{l}\right)$, запишем систему (5) в виде

$$
\begin{aligned}
& \dot{\varphi}=\omega+\varepsilon a(\varepsilon)+\Phi(\varphi, y, \bar{y}, v, \varepsilon), \\
& \dot{y}=(i \alpha+\varepsilon b(\varepsilon)) \cdot y+Y(\varphi, y, \bar{y}, v, \varepsilon), \\
& \dot{\bar{y}}=(-i \alpha+\varepsilon \bar{b}(\varepsilon)) \cdot \bar{y}+\bar{Y}, \\
& \dot{v}=Q v+V(\varphi, y, \bar{y}, v, \varepsilon),
\end{aligned}
$$


где $a(\varepsilon), b(\varepsilon)$ - векторы, зависящие только от $\varepsilon$.

Определим натуральное число $\beta$ с помощью равенства $\varepsilon \operatorname{Re} b(\varepsilon)=\varepsilon^{\beta} c(\varepsilon)$, где $c(0) \neq 0$. Пусть в неравенствах (6), которым по определению удовлетворяет система $(11), \nu \geqslant \beta+1$ (если в системе (11) последнее уравнение отсутствует, достаточно предположить, что $\nu \geqslant \beta)$. Предположим, что неравенство (3) выполняется в более широкой, чем в п. 2 области измерения чисел $p_{j}$, а именно при

$$
|q|>0, \quad|p| \leqslant 4, \quad\left|p_{1}+\cdots+p_{2 l}\right| \leqslant 3 .
$$

Лемма 2. При сделанных предположсениях существует замена

$$
\begin{aligned}
& \varphi=\psi+F(\psi, z, \bar{z}, \varepsilon), \\
& y=z+G(\psi, z, \bar{z}, \varepsilon), \quad \bar{y}=\bar{z}+\bar{G}, \\
& v=x+H(\psi, z, \bar{z}, \varepsilon),
\end{aligned}
$$

әде $\psi \in T^{m}, F, G, H$ - полиномы по $z, \bar{z}$, причем разложение $F$ по координатам $z, \bar{z}$ начинается с членов первого порядка, а разложсения $G, \bar{G}$ и $H$ - со второго, переводящее систему (11) в систему

$$
\begin{aligned}
\dot{\psi} & =\omega+\varepsilon a(\varepsilon)+A(z \cdot \bar{z})+\Psi(\psi, z, \bar{z}, x, \varepsilon), \\
\dot{z} & =(i \alpha+\varepsilon b(\varepsilon)+M(z \cdot \bar{z})) \cdot z+Z(\psi, z, \bar{z}, x, \varepsilon), \\
\dot{\bar{z}} & =(-i \alpha+\varepsilon \bar{b}(\varepsilon)+\bar{M}(z \cdot \bar{z})) \cdot \bar{z}+\bar{Z}, \\
\dot{x} & =Q x+X(\psi, z, \bar{z}, x, \varepsilon),
\end{aligned}
$$

где $A, M-$ постоянные матрицы,

$$
\begin{aligned}
& \Psi\left(\psi, \varepsilon^{\beta / 2} z, \varepsilon^{\beta / 2} \bar{z}, 0, \varepsilon\right)=O\left(\varepsilon^{\beta+1 / 2}\right), \\
& Z\left(\psi, \varepsilon^{\beta / 2} z, \varepsilon^{\beta / 2} \bar{z}, 0, \varepsilon\right)=O\left(\varepsilon^{3 \beta / 2+1}\right), \\
& X\left(\psi, \varepsilon^{\beta / 2} z, \varepsilon^{\beta / 2} \bar{z}, 0, \varepsilon\right)=O\left(\varepsilon^{3 \beta / 2+1 / 2}\right) .
\end{aligned}
$$

Лемма 2 доказьвается аналогично лемме 1 (см. [2], [3]) с учетом неравенства (6).

Введем в системе (14) полярные координаты $\rho, \tau$ по формулам

$$
z=\rho \cdot e^{i \tau}, \quad \bar{z}=\rho \cdot e^{-i \tau}, e^{i \tau}=\left(e^{i \tau_{1}}, \ldots, e^{i \tau_{m}}\right) .
$$

Получим систему

$$
\begin{aligned}
\dot{\psi} & =\omega+\varepsilon a(\varepsilon)+A \rho^{2}+\widetilde{\Psi}(\psi, \tau, \rho, x, \varepsilon), \\
\dot{\tau} \cdot \rho & =\left(\alpha+\varepsilon a(\varepsilon)+D \rho^{2}\right) \cdot \rho+T(\psi, \tau, \rho, x, \varepsilon), \\
\dot{\rho} & =\left(\varepsilon^{\beta} c(\varepsilon)+C \rho^{2}\right) \cdot \rho+R(\psi, \tau, \rho, x, \varepsilon), \\
\dot{x} & =Q x+\widetilde{X}(\psi, \tau, \rho, x, \varepsilon),
\end{aligned}
$$

где $\rho^{2}=\rho \cdot \rho, d=\operatorname{Im} b, D=\operatorname{Im} M, C=\operatorname{Re} M$. Из леммы 2 и (15) вытекает, что функции $\widetilde{\Psi}, T, R, \widetilde{X}$ удовлетворяют при $\varepsilon \rightarrow 0$ условиям

$$
\begin{aligned}
& \widetilde{\Psi}\left(\psi, \tau, \varepsilon^{\beta / 2} \rho, 0, \varepsilon\right)=O\left(\varepsilon^{\beta+1 / 2}\right), \\
& T\left(\psi, \tau, \varepsilon^{\beta / 2} \rho, 0, \varepsilon\right)=O\left(\varepsilon^{3 \beta / 2+1}\right), \\
& R\left(\psi, \tau, \varepsilon^{\beta / 2} \rho, 0, \varepsilon\right)=O\left(\varepsilon^{3 \beta / 2+1}\right), \\
& \widetilde{X}\left(\psi, \tau, \varepsilon^{\beta / 2} \rho, 0, \varepsilon\right)=O\left(\varepsilon^{3 \beta / 2+1 / 2}\right) .
\end{aligned}
$$

Предположим, что в системе (16)

$$
\operatorname{det} C \neq 0, \quad C^{-1} c(0)=\eta^{2}>0,
$$

где символ $\eta^{2}>0$ обозначает положительность всех координат вектора $\eta^{2}=\eta \cdot \eta$. 
ТЕорема 2. Если неравенства (3) имеют место в области (12), выполняется условие (18) и матрича $(\operatorname{diag} \eta) C(\operatorname{diag} \eta)$ некритична, то при $\beta \geqslant 2$ система (1) имеет при достаточно малых $\varepsilon>0$ инвариантный $(m+l)$-мерный тор.

ДокАЗАтЕЛЬСтво. Рассмотрим бифуркационное уравнение

$$
\varepsilon^{\beta} c(\varepsilon)+C \rho^{2}=0
$$

Решая его относительно $\rho^{2}$, находим $\rho^{2}=\varepsilon^{\beta} \eta^{2}(\varepsilon)$, где в силу $(18) \eta^{2}(0)=\eta^{2}>0$. Положим

$$
\rho=\varepsilon^{\beta / 2}(\eta(\varepsilon)+\sqrt{\varepsilon} r), \quad v=\varepsilon^{3 \beta / 2} w .
$$

В силу (17) в переменных $\psi, \tau, r, w$ система (16) имеет вид

$$
\begin{aligned}
\dot{\psi} & =\omega+\varepsilon \delta(\varepsilon)+\varepsilon^{\beta+1 / 2} \Psi^{*} \\
\dot{\tau} & =\alpha(\varepsilon)+\varepsilon^{\beta+1 / 2} T^{*} \\
\dot{r} & =\varepsilon^{\beta} S(\varepsilon) r+\varepsilon^{\beta+1 / 2} R^{*} \\
\dot{w} & =Q w+\sqrt{\varepsilon} W
\end{aligned}
$$

где $S(\varepsilon)=2(\operatorname{diag} \eta(\varepsilon)) C(\operatorname{diag} \eta(\varepsilon)), \Psi^{*}, T^{*}, R^{*}, W$ - гладкие функции при $\|r\|<r^{*}$, $\|w\|<w^{*},(\psi, \tau) \in T^{m+l}$.

Так как по условиям теоремы матрица $S(0)$ некритична, применима лемма 2.1 работы [4] (или ее обобщение в работах [2], [3]). Следовательно, система $(20)$ имеет $2 \pi$-периодическое по $\psi, \tau$ интегральное многообразие $r=r(\psi, \tau, \sqrt{\varepsilon}), w=w(\psi, \tau, \sqrt{\varepsilon})$. Подставляя эти равенства в (19) и учитывая $(15),(13),(4)$, получим утверждение теоремы.

ЗАмЕчАнИЕ. Пусть вьполняется условие (10). Тогда по теореме 1 система (1) имеет при $\varepsilon>0$ инвариантные торы как размерности $m$, так и размерности $m+l$. В таком случае говорят, что имеет место бифуркация тора $T^{m}$ с ответвлением $(m+l)$-мерного тора.

Случай $\beta=1$ был рассмотрен в [2], [3]. Там показано также, как получить условия существования инвариантных торов любой размерности, большей $m$. В случае $m=0$, $\beta=1, C=0$ условия существования устойчивых инвариантных торов размерности $l$ получены в работе [8].

В п. 1, 2 мы рассмотрели вопрос о сохранении и бифуркации инвариантного тора в алгебраических случаях. В этих случаях вопрос о существовании искомых торов решался методами теории Крылова-Боголюбова. При исследовании трансцендентных случаев необходимо использовать методы КАМ-теории. Соответственно удается доказать сохранение только таких инвариантных торов, движения на которых квазипериодические с базисными частотами $\omega_{1}, \ldots, \omega_{m}$. Некоторые трансцендентные случаи рассматриваются в следующем пункте.

4. Трансцендентный случай. Начнем с того, что укажем класс систем, для которых имеет место трансцендентньй случай. Пусть в системе (1) вектор $x$ четномерньй. Соответственно, вектор $\eta$ в системе (2) тоже четномерный. Матрицу $P$ в $(2)$ можно представить в виде $P=\left(P_{1}, \bar{P}_{1}\right)$, где $P_{1}=\operatorname{diag}\left(i \alpha_{1}, \ldots, i \alpha_{l}\right)$, а черта, как и выше, знак комплексной сопряженности. Следовательно, $\bar{P}_{1}=-P_{1}$. Предположим, что и 
собственные числа матрицы $Q$ разбиваются на две группы, отличающиеся лиш знаком. Тогда $Q=\left(Q_{1},-Q_{1}\right)$. Система (2) примет вид

$$
\begin{aligned}
\dot{\theta} & =\omega+\Theta, \\
\dot{\xi}_{1} & =P_{1} \xi_{1}+\Xi_{1}, \quad \dot{\xi}_{2}=-P_{1} \xi_{2}+\Xi_{2}, \\
\dot{\eta}_{1} & =Q_{1} \eta_{1}+H_{1}, \quad \dot{\eta}_{2}=-Q_{1} \eta_{2}+H_{2} .
\end{aligned}
$$

Пусть, далее,

$$
\begin{aligned}
\Theta\left(-\theta, \xi_{2}, \xi_{1}, \eta_{2}, \eta_{1}\right) & =\Theta\left(\theta, \xi_{1}, \xi_{2}, \eta_{1}, \eta_{2}\right), \\
\Xi_{1}\left(-\theta, \xi_{2}, \xi_{1}, \eta_{2}, \eta_{1}\right) & =-\Xi_{2}\left(\theta, \xi_{1}, \xi_{2}, \eta_{1}, \eta_{2}\right), \\
H_{1}\left(-\theta, \xi_{2}, \xi_{1}, \eta_{2}, \eta_{1}\right) & =-H_{2}\left(\theta, \xi_{1}, \xi_{2}, \eta_{1}, \eta_{2}\right)
\end{aligned}
$$

При выполнении условий (22) систему (21) будем называть обратимой.

Нетрудно убедиться, что для обратимых систем всегда имеет место трансцендентный случай. Действительно, из формул (7) и (8) вытекает, что система (5) тоже является обратимой. В силу (22) матрицы $B_{i}$ в системе (5) отличаются от своих комплексно сопряженных лишь знаком, т.е. все их элементы - чисто мнимые числа. Следовательно, условие (10) не выполняется ни при каком натуральном $\nu$.

Далее мы ограничимся рассмотрением случая $n=2 l$, т.е. случая, когда в системе (1) некритические переменные отсутствуют. Кроме того, предположим, что функции в правой части (1) вещественно аналитичны при достаточно малых $\|x\|,|\varepsilon|,\|\operatorname{Im} \theta\|$.

Выполним замену (4) при $\nu=1$. Система (9) примет вид

$$
\begin{aligned}
& \dot{\varphi}=\omega+\varepsilon a+\varepsilon^{3 / 2} \Phi^{*}, \\
& \dot{z}=(P+\varepsilon B) z+\varepsilon^{3 / 2} Z,
\end{aligned}
$$

где $B=\operatorname{diag}\left(B_{1}, \ldots, B_{2 l}\right)$, функции $\Phi^{*}, Z$ аналитичны при достаточно малых $\|z\|,|\sqrt{\varepsilon}|$, $\|\operatorname{Im} \varphi\|$. Предположим, что

$$
B_{j}=i \beta_{j} \neq 0, \quad j=1, \ldots, 2 l,
$$

где $\beta_{j}$ вещественны и попарно различны.

Числа $\lambda_{j}=i \alpha_{j}, B_{j}=i \beta_{j}$ распадаются на комплексно сопряженные пары. Пусть $\alpha_{k}=-\alpha_{k+l}, \beta_{k}=-\beta_{k+l}, k=1, \ldots, l$. Не теряя общности, считаем, что $\beta_{k}>0$, $k=1, \ldots, l$.

Рассмотрим числа $\mu_{1}, \ldots, \mu_{2 l}, \mu_{k}=-\mu_{k+l}, k=1, \ldots, l$, удовлетворяющие условию (3) при $K \varepsilon=\gamma$, т.е. условию

$$
\left|\sum_{i=1}^{m} q_{i} \omega_{i}+\sum_{j=1}^{2 l} p_{j} \mu_{j}\right|>K \varepsilon|q|^{-\tau}, \quad K>0, \quad \tau>0
$$

где $|q|>0,|p| \leqslant 2,\left|p_{1}+\cdots+p_{2 l}\right| \leqslant 1$.

Образуем матрицу $M=\operatorname{diag}\left(i \mu_{1}, \ldots, i \mu_{2 l}\right)$. В работе [5] доказано следующееутверждение. 
Лемма 3. Существует число $\varepsilon^{*}(K)>0$, обладающее свойством: существуют векторные функиии $b(\psi, \sqrt{\varepsilon}), c(\psi, \sqrt{\varepsilon}), s(\sqrt{\varepsilon})$ и матричные функиии $C(\psi, \sqrt{\varepsilon})$, $\Delta(\sqrt{\varepsilon})$, аналитические при $\|\operatorname{Im} \psi\|<\psi^{*},|\sqrt{\varepsilon}|<\sqrt{\varepsilon^{*}}$ и такие, что замена

$$
\begin{aligned}
& \varphi=\psi+\varepsilon^{3 / 2} b(\psi, \sqrt{\varepsilon}) \\
& z=\zeta+\varepsilon^{3 / 2} c(\psi, \sqrt{\varepsilon})+\varepsilon^{3 / 2} C(\psi, \sqrt{\varepsilon}) \zeta
\end{aligned}
$$

приводит систему

$$
\begin{aligned}
& \dot{\varphi}=\omega+\varepsilon^{3 / 2} s+\varepsilon^{3 / 2} \Phi^{*} \\
& \dot{z}=\left(M+\varepsilon^{3 / 2} \Delta\right) z+\varepsilon^{3 / 2} Z
\end{aligned}
$$

$\kappa в u \partial y$

$$
\begin{aligned}
& \dot{\psi}=\omega+F(\psi, \zeta, \sqrt{\varepsilon}) \\
& \dot{\zeta}=M \zeta+G(\psi, \zeta, \sqrt{\varepsilon}),
\end{aligned}
$$

$2 \partial e$

$$
F(\psi, 0, \sqrt{\varepsilon})=0, \quad G(\psi, 0, \sqrt{\varepsilon})=0, \quad \frac{\partial G}{\partial \zeta}(\psi, 0, \sqrt{\varepsilon})=0
$$

При этом $\Delta$ - диагональная матрииа, и комплексно сопряэсенным әлементам матрицы $M$ соответствуют комплексно сопряжсенне әлементы матрицы $\Delta$.

Рассмотрим вопрос о существовании чисел $\mu_{1}, \ldots, \mu_{2 l}$, удовлетворяющих условию (25). Примем $\tau=m+1$. Положим $\mu_{k}-\alpha_{k}=\varepsilon_{k}, k=1, \ldots, l$. При $|p|=0$ неравенство (25) выполняется в силу (3), так как можно считать, что $K \varepsilon<\gamma$. При $\|p\|=1$ неравенство (25) имеет вид

$$
\left|\sum_{i=1}^{m} q_{i} \omega_{i}-\left(\alpha_{k}+\varepsilon_{k}\right)\right|>K \varepsilon|q|^{-(m+1)}, \quad k=1, \ldots, l .
$$

При $|p|=2$ неравенство (25) принимает вид

$$
\left|\sum_{i=1}^{m} q_{i} \omega_{i}+\left(\alpha_{j}+\varepsilon_{j}\right) \mp\left(\alpha_{k}+\varepsilon_{k}\right)\right|>K \varepsilon|q|^{-(m+1)} .
$$

Следующая лемма доказьвается стандартными рассуждениями (см. [6]).

Лемма 4. Для любых $\delta \in(0,1), \sigma>0$ мохнно указать $K(\delta, \sigma)>0$ так, чтобъ множество $\Gamma$, образованное числами $\Omega$, для которых при фиксированных $\omega_{i}$ выполняется неравенство

$$
\left|\sum_{i=1}^{m} q_{i} \omega_{i}-\Omega\right|>K \varepsilon|q|^{-(m+1)}
$$

обладало следующим свойством: для любого интервала L длины $2 \sigma \varepsilon$ выполняется неравенство $\operatorname{mes}(\Gamma \cap L)>2 \sigma \varepsilon(1-\delta)$. 
СлЕДСТВИЕ. При любых $\sigma_{k}>0, k=1, \ldots, l$, неравенства (30) и (31) выполняются для $\epsilon_{k} \in \Gamma_{k} \subset\left(-\sigma_{k} \varepsilon, \sigma_{k} \varepsilon\right)$, причем за счет выбора $K$ можно добиться, чтобб мера множества $\Gamma_{k}$ была сколь угодно близкой к величине $2 \sigma \varepsilon$.

Предположим, что выполняются условия

$$
\Delta(\sqrt{\varepsilon})=i d(\sqrt{\varepsilon})
$$

где $d(\sqrt{\varepsilon})$ - вещественно аналитическая при $|\sqrt{\varepsilon}|<\sqrt{\varepsilon^{*}}$ матричная функция, и

$$
a=s(\sqrt{\varepsilon})=0 .
$$

Теорема 3. Если выполняются условия (3), (24), (32), (33), то в любом интервале $\left(0, \varepsilon_{0}\right)$ существуют значения $\varepsilon$, которым соответствуют инвариантные торы $T_{\varepsilon}^{m}$ системы (1), причем $T_{\varepsilon}^{m} \rightarrow T^{m}$ при $\varepsilon \rightarrow 0$. Более того, мера множества, образованного числами $\varepsilon$, обладающими указанным свойством, әквивалентна как бесконечно малая величина $\varepsilon_{0}$. Движения на торах $T_{\varepsilon}^{m}$ являются квазипериодическими с базисными частотами $\omega_{1}, \ldots, \omega_{m}$.

ДокаЗАтельСтво. Рассмотрим систему (28). Из (29) вытекает, что она имеет решение $\psi=\omega t, \zeta=0$, которому в силу $(26)$ соответствует решение

$$
\varphi=\omega t+\varepsilon^{3 / 2} b(\omega t, \sqrt{\varepsilon}), \quad z=\varepsilon^{3 / 2} C\left(\omega t+\varepsilon^{3 / 2} b, \sqrt{\varepsilon}\right)
$$

системы (27). Система (27) совпадает в силу (33) с интересующей нас системой (23), если

$$
P+\varepsilon B=M+\varepsilon^{3 / 2} \Delta(\sqrt{\varepsilon}) .
$$

В неравенстве (25) зафиксируем $\varepsilon=\varepsilon^{*}(K)$, считая $K$ достаточно малой величиной. Пусть $\varepsilon \in\left(0, \varepsilon_{0}\right), \varepsilon_{0}=\frac{1}{2} \varepsilon^{*}, \sigma_{k}=\frac{1}{2} \beta_{k}$. Зафиксируем $K$, обеспечивающее согласно следствию из леммы 4 достаточную близость меры множеств $\Gamma_{k}$ к величинам $2 \sigma_{k} \varepsilon_{0}$ при всех $k=1, \ldots, l$. Так как матрица $M$ зависит от параметров $\varepsilon_{1}, \ldots, \varepsilon_{l}$, то функция $\Delta$ зависит не только от $\sqrt{\varepsilon}$, но и от этих параметров. Как функция $\varepsilon_{1}, \ldots, \varepsilon_{l}$ она определена в силу $(30)$ и (31) и следствия из леммы 4 на некотором множестве $\Gamma \subset \prod_{k=1}^{l}\left(-\sigma_{k} \varepsilon_{0}, \sigma_{k} \varepsilon_{0}\right)$. Более того, она может быть продолжена до функции класса $C^{\infty}$ на прямое произведение интервалов $\left(-\sigma_{k} \varepsilon_{0}, \sigma_{k} \varepsilon_{0}\right)[7]$. Продолженную функцию обозначим

$$
E=i \operatorname{diag}\left(E_{1}, \ldots, E_{l},-E_{1}, \ldots,-E_{l}\right) .
$$

Распишем уравнение (35) с продолженной функцией $\Delta$ покоординатно:

$$
\beta_{k} \varepsilon-\varepsilon_{k}-\varepsilon^{3 / 2} E_{k}\left(\sqrt{\varepsilon}, \varepsilon_{1}, \ldots, \varepsilon_{l}\right)=0, \quad k=1, \ldots, l .
$$

Якобиан этой системы по $\varepsilon_{1}, \ldots, \varepsilon_{l}$ при $\varepsilon=\varepsilon_{1}=\cdots=\varepsilon_{l}=0$ отличен от нуля. Следовательно, система (36) имеет решение $\varepsilon_{k}=\beta_{k} \varepsilon+f_{k}(\varepsilon), k=1, \ldots, l$, где $f_{k}=o(\varepsilon)$. $\Phi$ ункции $f_{k}$ определены при $\varepsilon \in\left(0, \varepsilon_{0}\right)$. Однако нас интересуют лишь те значения $\varepsilon$, которым соответствуют

$$
\varepsilon_{k}=\beta_{k} \varepsilon+f_{k}(\varepsilon) \in \Gamma_{k}, \quad k=1, \ldots, l .
$$


По следствию из леммы 4 каждое из этих условий определяет множество значений $\varepsilon \in\left(0, \varepsilon_{0}\right)$, мера которого сколь угодно близка к величине $\varepsilon_{0}$. Следовательно, мера множества значений $\varepsilon$, удовлетворяющих условиям (37), также сколь угодно близка к $\varepsilon_{0}$. При указанных значениях $\varepsilon$ система (23) имеет решение $(34)$, которому соответствует искомый инвариантньй тор системы (1). Теорема 3 доказана.

Условие (3) при фиксированной матрице $P$ вьполняется для почти всех векторов частот $\omega$. В противоположность этому условия (32), (33) налагают существенные ограничения на рассматриваемую систему. Условие (32) соответствует ограничениям, которые налагает на систему требование наличия трансцендентного случая. По крайней мере, для обратимьх систем (21), (22), для которых, как было показано, всегда имеет место трансцендентньй случай, выполняется и условие (32), так как преобразование (26) получается как композиция бесконечного числа преобразований, каждое из которых оставляет систему обратимой. Что касается условия (33), то оно вьполняется автоматически, если частоты тора $T^{m}$ не подвергаются возмущениям. В этом случае речь идет о системе, правая часть которой является квазипериодической функцией времени с вектором $\omega$ базисных частот. В частности, полученные результаты применимы к квазилинейной системе

$$
\dot{x}=P x+\varepsilon X(t, x, \varepsilon),
$$

где $X$ - квазипериодическая функция $t$, если эта система является либо гамильтоновой, либо обратимой (подробности см. в работе [6]).

Санкт-Петербургский государственный университет

Поступило

10.02 .95

\section{СПИСОК ЦИТИРОВАННОЙ ЛИТЕРАТУРЫ}

[1] Боголюбов Н.Н., Митропольский Ю.А., Самойленко А. М. Метод ускорений сходимости в нелинейной механике. Киев: Наукова думка, 1969.

[2] Бибиков Ю. Н. Многочастотные нелинейные колебания и их бифуркации. Л.: Изд-во ЛГУ, 1991.

[3] Бибиков Ю.Н. Построение инвариантных торов систем дифференциальных уравнений с малым параметром // Тр. ЛМО. 1991. Т. 1. С. 26-53.

[4] Hale J. K. Integral manifolds of perturbed differential systems // Ann. of Math. 1961. V. 73. P. 496-531.

[5] Moser J. K. Convergent series expansions for quasiperiodic motions // Math. Ann. 1967. V. 169. P. 136-176.

[6] Бибиков Ю.Н. О существовании квазипериодических движений и квазилинейньх систем // ПММ. 1995. Т. 59. №1. C. 23-31.

[7] Broer H. W., Huitema G. B., Takens F. Unfolding of quasiperiodic tori // Mem. Amer. Soc. 1990. V. 83. P. 1-82.

[8] Бибиков Ю.Н.Бифуркация устойчивого инвариантного тора из состояния равновесия // Матем. заметки. 1990. Т. 48. №1. С. 15-19. 